\title{
SARCOIDOSIS OF HANDS
}

\author{
Patrick FitzGerald and F. O. C. Meenan, Dublin, Ireland
}

From St Vincent's Hospital, Dublin

The various manifestations of the complex disease known as sarcoidosis have only been recognised in recent years. The stages in its recognition have been well summarised by Gray (1941). In 1889 Besnier described a symmetrical granulomatous condition of the nose, ears and fingers which he called "lupus pernio." In 1899 Boeck reported a skin disorder characterised by the appearance on the face of granulomatous swellings resembling lupus vulgaris, and coined the name " cutaneous sarcoid." It was recognised in 1898 by Hutchinson, who called it "Mortimer's malady" after the patient concerned. In 1917 Schaumann showed that these conditions were variants of the same disease and merely cutaneous manifestations of a disorder affecting many organs such as the lymphatic system, the bone marrow, the lungs and other viscera.

According to Ellis (1940), Jüngling in 1920 reported sarcoid lesions of the bones of the extremities under the title " osteitis tuberculosa multiplex cystica." Later he substituted the word " cystoides" for " cystica," because the lesions are in fact solid and only appear cystic in the radiographs. Ellis emphasised that Jüngling's disease is not cystic tuberculosis of bone, and that the radiographic appearance is due to replacement of bone and marrow by sarcoid material.

McCort, Wood, Hamilton and Ehrlich (1947), in a series of twenty-seven cases of sarcoid, found six with lesions in the hands, though none of these patients had any symptom referable to the bony changes. The osseous involvement was of two types: one the condition described by Jüngling, and the other a diffuse rarefaction with reduction in the number of bony trabeculae in the medulla and slight thinning of the cortex. Riley (1950), in a series of fifty-two cases, reported osseous lesions in the extremities in 13 per cent, mainly in the phalanges. The lesions were either localised punched-out " cystic " areas without evidence of surrounding inflammatory reaction, or a striking alteration of the trabecular structure of the bone with extensive cortical atrophy and resorption, as in the present case. A coarsely reticulated appearance of the medulla was characteristic. Holt and Owens (1949) found nine cases of bone involvement in fifty-five cases. They regarded the middle and distal phalanges of the hands and feet as the commonest sites of bone lesions. Longcope and Freiman (1952) found nineteen bone lesions in 100 cases. Mather (1957) reported that in a recent survey, with control radiographs, bone changes were found in only nine out of the 120 patients. He suggested that the lower frequency in this series may be because most of the patients had predominantly intrathoracic sarcoidosis; there were only thirteen cases of skin sarcoid, of which five showed digital bone disease. Sarcoid foci had in fact been reported in almost every bone, but they are of diagnostic value only in the hands and feet.

When sarcoid is present in the marrow, secondary changes appear in the adjacent bone in one of several patterns. If the disease progresses slowly, lacunar resorption occurs over a relatively large area, but most intensely at the distal ends of the proximal and middle phalanges, where localised cavities form. In addition, perivascular infiltration of Haversian canals may occur, with consequent thinning of the cortex and destruction of the finer trabeculae. In the very early stages of the disease there is non-specific osteoporosis which is later supplanted by a lace-work pattern of bone destruction-the commonest and most specific radiological manifestation of osseous sarcoid.

Calcium metabolism-Mather (1957) stated that hypercalcaemia occurs so infrequently in patients with sarcoidosis not receiving vitamin $D$ that routine estimation of the serum calcium 
is unnecessary. He reported nine cases with bone lesions and no upset of calcium metabolism. In the case of bone sarcoidosis reported here the first serum calcium estimation by the Kramer and Tisdall method, 13.5 milligrams per cent, was well above normal, though it became a low normal figure, $9 \cdot 3$ milligrams per cent, before calciferol therapy was started.

\section{CASE REPORT}

A typist then aged twenty-two years was first seen by us in February 1951. In 1943, when she was fourteen years old and otherwise healthy she began to notice burning pain and swelling in the middle finger of the left hand. In 1944 the finger was amputated through the middle phalanx. In 1945 the same condition began in the corresponding finger of the right hand, and it too was partially amputated in 1946. Other regions of both hands became involved to a somewhat lesser extent, as shown in Figure 1.

The patient said that the condition began in each finger with a burning pain which lasted four or five days. Then the pain would subside and the swelling remain. If the tip of a finger
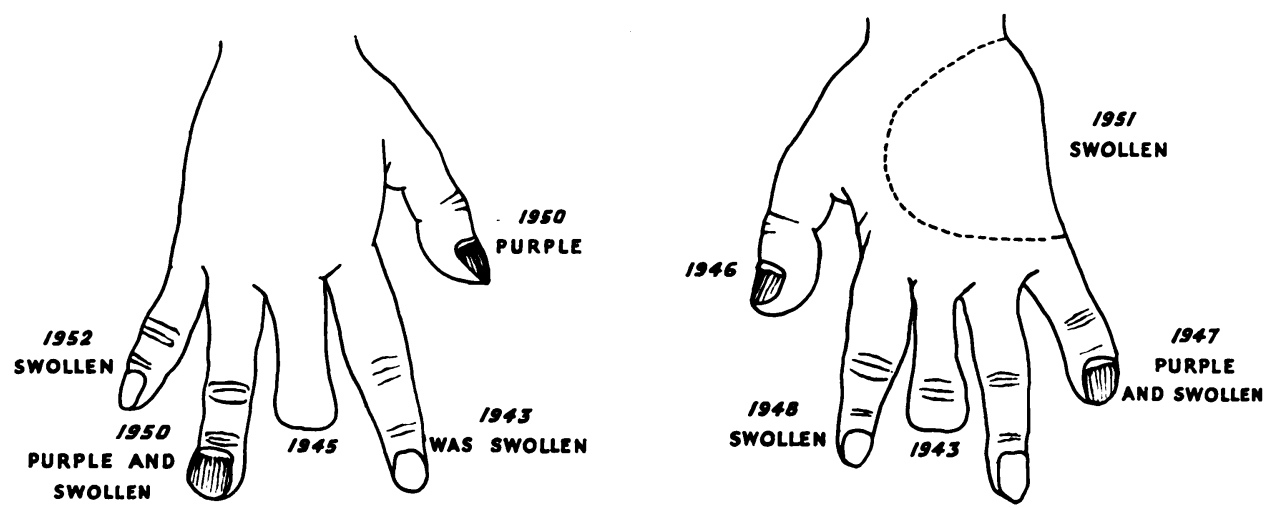

Fig. 1

Diagram showing progression of the disease. All dates refer to onset.

were involved the nail became wider than normal and sometimes became brittle and striated longitudinally; if the base of a finger were involved the nail was not affected. Her hands became tired if she worked much, and because of the amputations she was no longer able to use a typewriter. The feet were not affected. She had no chest trouble and there was no family history of tuberculosis.

At the time of examination she complained of burning pain and swelling in the thumb, index finger, little finger and ulnar area of the back of the left hand, and in the thumb, ring and little fingers of the right hand. The affected skin was reddish-blue and in the swollen regions the skin was shiny and smooth (Fig. 2). The tips of the remaining affected fingers were bulbous. The amputated fingers were also bulbous and the tips of the stumps were a little blue. The ulnar part of the back of the left hand was oedematous. Movement of the fingers was free. There was no sign of peripheral nerve involvement and the circulation in both hands appeared normal. There were no other clinical signs of sarcoid elsewhere in the body.

From inquiries it was clear that the condition had originally been treated as a fungus infection, though there had been no purulent discharge except for a short time from the right ring finger. The opportunity for biopsy afforded by the amputations had been missed.

It was felt that the condition was most likely to be Boeck's sarcoid affecting the fingers. Because the patient lived in the country it was only possible to see her at infrequent intervals. The investigations were not complete until June 1951, when the diagnosis was established mainly from biopsy of the skin and from the radiographic appearance of the fingers. 
Investigations-The Mantoux test, the Wassermann reaction, the sedimentation rate, the blood count and a search for fungus infection gave no positive information. A number of tests more specifically related to sarcoidosis gave the following results:

Serum protein-Total protein was 7.5 grammes per cent (globulin 3.9 grammes per cent;

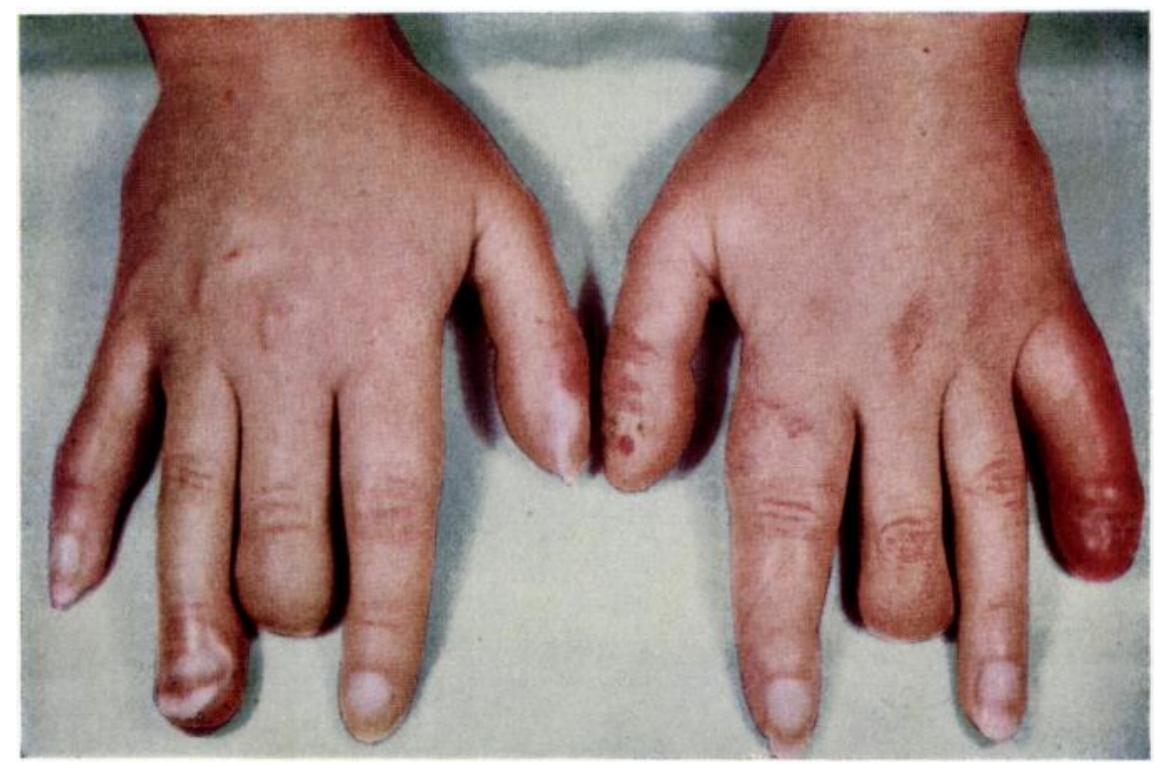

Fig. 2

Photograph of the hands in 1951, before treatment was begun.

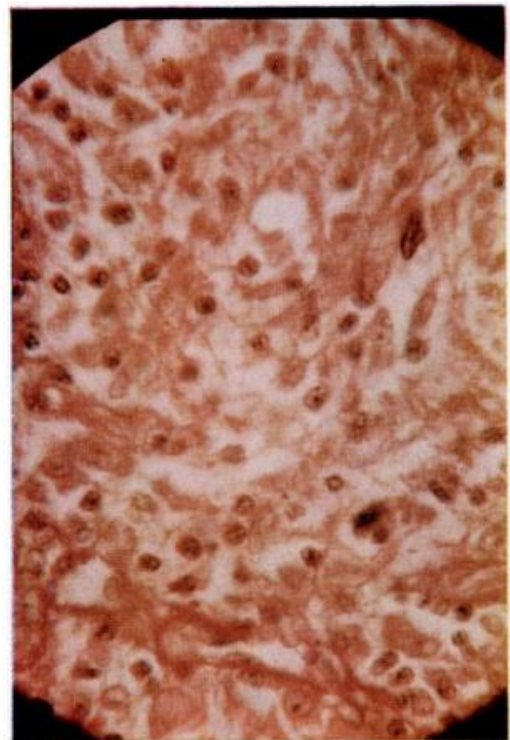

Fig. 3

Low-power and high-power photomicrographs of sections from the skin of the ulnar side of the left hand, obtained at biopsy.

albumen 3.6 grammes per cent). Blood calcium-(February 1951) 13.5 milligrams per 100 millilitres; (June 1951) 11.2 milligrams per 100 millilitres; (March 1952) $9 \cdot 3$ milligrams per 100 millilitres. Alkaline phosphatase-4.3 King-Armstrong units. Uveal tractNo sarcoid deposits seen on examination with slit lamp. 


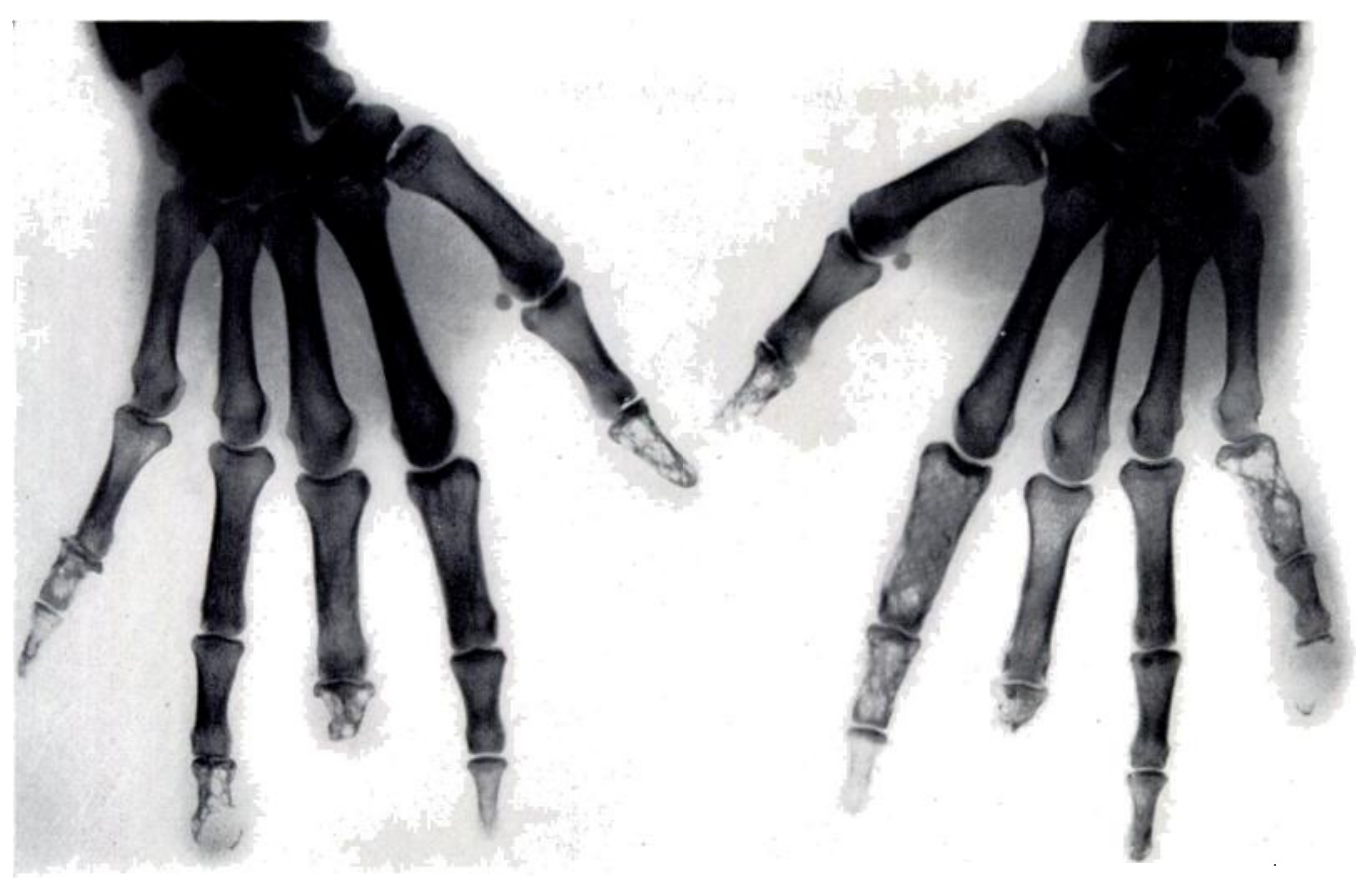

Fig. 5

Radiographs of the hands in 1951 , before treatment was begun.

Biopsy - Needle biopsy of an affected phalanx showed no sign of sarcoid. Skin biopsy from the ulnar side of the left hand ( Dr R. P. Towers) showed, in the dermis, several follicles of epitheloid cells, well demarcated, with only very few surrounding lymphocytes. There was no trace of caseation. The appearances were those of sarcoidosis (Figs. 3 and 4).

Radiographic examination-There was coarse expansion of the trabecular structure characteristic of Boeck's disease (Figs. 5 and 6). The pattern of bone damage closely followed the superficial changes except for the absence of bone change in relation to the swollen ulnar side of the left hand. There was almost complete disappearance of the phalanges in the tips of the severely involved right ring and left little fingers. Radiographs of the feet showed no bony changes. The radiograph of the chest showed a slight increase in striation, not typical of sarcoid.

Sarcoid material was not readily available at that time, so the Kveim test was not carried out.

Treatment-In June 1951 the patient was admitted to hospital and given 600 milligrams hydroxy-phenyl-cinchoninic acid a day for ten days. Three months later she reported that the hands felt better and were not so red. In March 1952 the renal function tests being satisfactory, 100,000 units of vitamin $D_{2}$ (calciferol) daily were prescribed. Because of nausea and vomiting, the dose was reduced to 50,000 units daily in the following month, and this dosage was continued for six months. Owing to the striking improvement (Fig. 7) treatment was then stopped.

When last seen by us in May 1955 the patient had no complaints and the hands looked quite healthy. The colour was

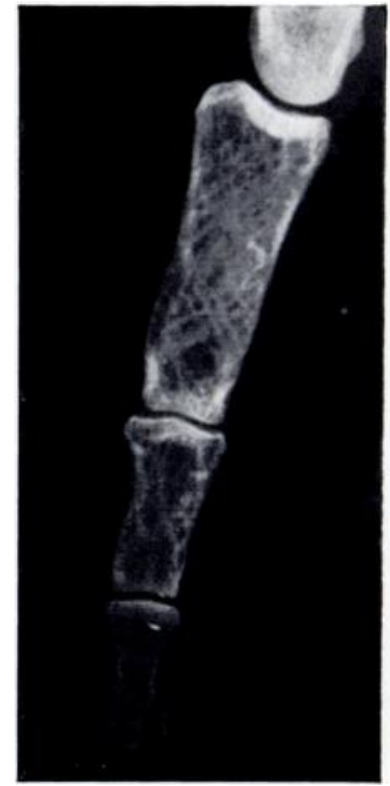

Fig. 6

Contact print from radiograph of left index finger showing the trabecular structure seen typically in Boeck's sarcoidosis. 


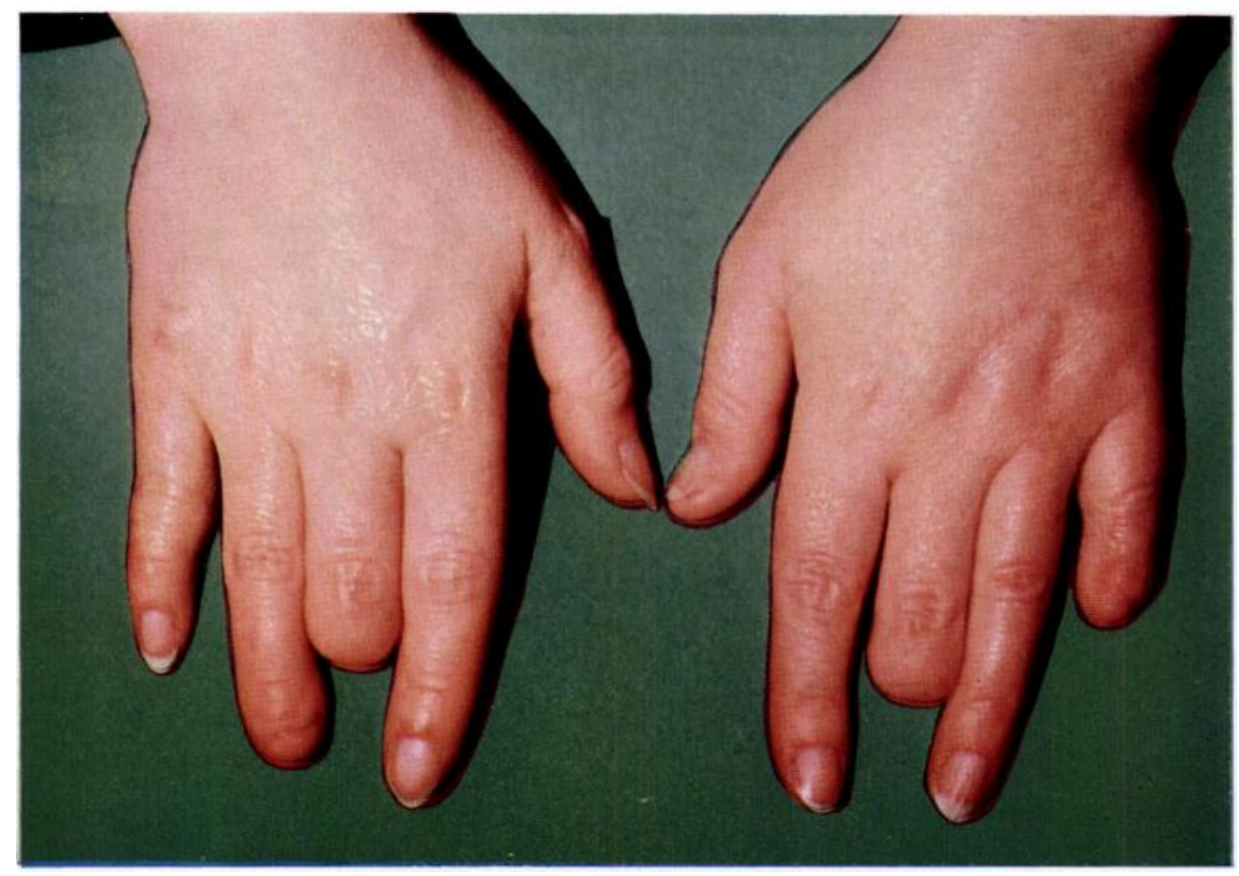

Fig. 7

Photograph of the hands at the completion of treatment.

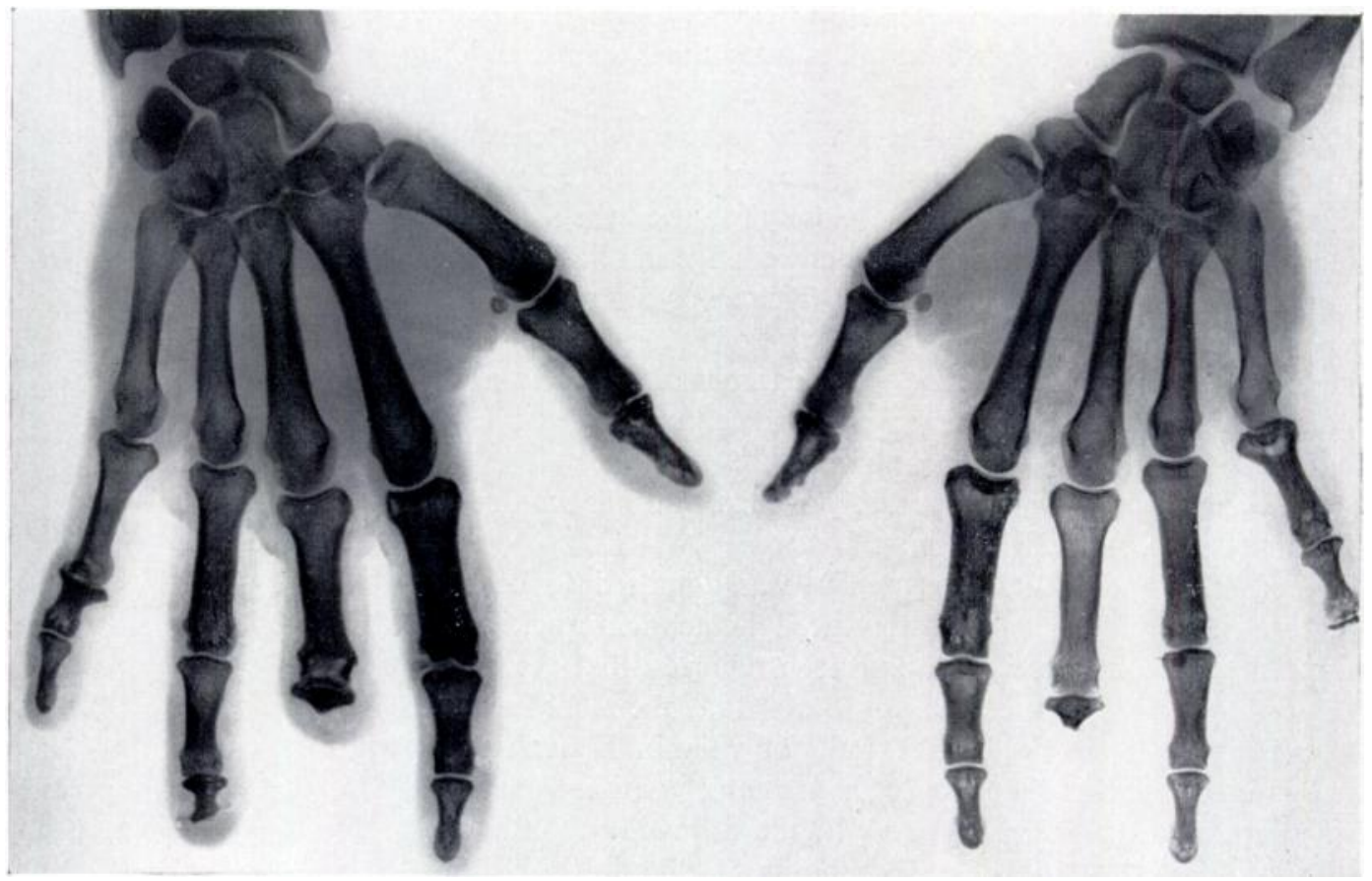

Fig. 8

Radiograph of the hands in 1957, five years after the completion of treatment. 
good, there was no swelling, and the nails had largely recovered. An exception was the left little finger, the tip of which had atrophied.

A radiograph taken in February 1957 (Fig. 8) showed the improvement in the bony structure. At the same time the patient wrote to say that her hands remained healthy and that she had resumed work as a typist.

\section{COMMENT}

Failure to suspect this unusual but typical condition led to the unnecessafy partial amputation of two fingers in a typist. The indolent history, the pain, the spread up the digits, the asymmetry of the involvement and the absence of pus should have brought sarcoidosis into consideration. Biopsy and radiography should have established the diagnosis.

The treatment of this patient raised some problems. Because she lived in the country it was not possible to keep her under constant supervision. The short course of hydroxyphenyl-cinchoninic acid appeared to improve the condition slightly, but it was not a drug that could be given safely for long. By March 1952 calciferol appeared to be the drug of choice and there seems to be little doubt that it was effective.

Goldsmith and Hellier (1954), summarising the results of other workers who have treated sarcoidosis with calciferol, state that opinions differ about its effect. On the one hand Dowling et al. (1948) saw improvement in six cases out of seven. On the other hand, Nelson (1949) saw no difference between eight patients treated with calciferol and eight controls, and Mather went so far as to state that complications are so frequent that its use should be discontinued.

When this case was under consideration, the place of cortisone and A.C.T.H. in the treatment of sarcoidosis had not been clearly defined, but it has now become clear that these drugs have a valuable suppressive action in sarcoidosis. Since the patient appeared to be responding well to calciferol, it was decided that there was no need to change her treatment. The improvement with calciferol appears to have been more than coincidence. It occurred promptly and has persisted for almost three years in a case in which symptoms had been troublesome for nearly ten years. This case, however, has been presented more particularly to illustrate the diagnostic features of sarcoidosis of the hands.

\section{REFERENCES}

BESNIER, E. (1889): Lupus pernio de la face; synovites fongueses (scrofulo-tuberculeuses) symétriques des extrémités supérieures. Annales de Dermatologie et de Syphiligraphie, 10, 333.

BOECK, C. (1899): Multiple Benign Sarkoid of the Skin. Journal of Cutaneous and Genito-Urinary Diseases, $17,543$. Dowling, G. B., Gauvain, S., and Macrae, D. E. (1948): Vitamin D in Treatment of Cutaneous Tuberculosis. British Medical Journal, $\mathbf{i}, 430$.

Ellis, F. A. (1940): Jüngling's “ ostitis tuberculosa multiplex cystoides" is not Cystic Tuberculous Osteitis. Acta Medica Scandinavica, 104, 221.

Goldsmith, W. N., and Hellier, F. F. (1954): Recent Advances in Dermatology. Second edition. London: J. \& A. Churchill Ltd.

Gray, A. M. H. (1941): Sarcoidosis. In A Textbook of the Practice of Medicine, edited by F. W. Price. Sixth edition, p. 1,476. London: Humphrey Milford, Oxford University Press.

Holt, J. F., and Owens, W. I. (1949): The Osseous lesions of Sarcoidosis. Radiology, 53, 11.

Hutchinson, J. (1898): Cases of Mortimer's Malady (Lupus Vulgaris Multiplex non-ulcerans et non-serpiginosus). Archives of Surgery, London, 9, 307.

JüNGLING, O. (1920): Ostitis tuberculosa multiplex cystica. Fortschritte auf dem Gebiete der Röntgenstrahlen, 27, 375.

Longcope, W. T., and Freiman, D. G. (1952): A Study of Sarcoidosis. Medicine, Baltimore, $31,1$.

MCCoRt, J. J., Wood, R. H., Hamilton, J. B., and Ehrlich, D. E. (1947): Sarcoidosis. Archives of Internal Medicine, 80, 293.

Mather, G. (1957): Calcium Metabolism and Bone Changes in Sarcoidosis. British Medical Journal, i, 248. Nelson, C. T. (1949): Calciferol in the Treatment of Sarcoidosis. Journal of Investigative Dermatology, $13,81$. Riley, E. A. (1950): Boeck's Sarcoid. American Review of Tuberculosis, 62, 231.

SchaumanN, J. (1917): Étude sur le lupus pernio et ses rapports avec les sarcoides et la tuberculose. Annales de Dermatologie et de Syphiligraphie, 5. série 6, 357.

VOL. $40 \mathrm{~B}$, NO. 2, MAY 1958

G 